
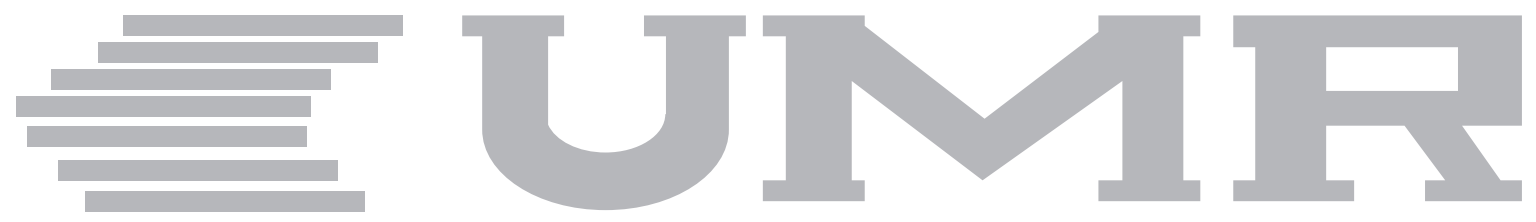

\title{
UPGTO MANAGEMENT REVIEW
}

Revista UPGTO Management Review 2021

\section{Vol.6 Núm.2}

\section{La internacionalización en las Instituciones de Educación Superior, caso UNITESBA Universidad}

Internationalization in Institutions Higher Education, UNITESBA case University

\author{
Patricia Troncoso, \\ Carmen King,
}

Recepción es 29 de abril de 2021 , Aceptación es 20 de agosto de 2021 .

No. de Reserva 04-2014-08 I I 025600-23

ISSN: 2007-977X 


\title{
La internacionalización en las Instituciones de Educación Superior, caso UNITESBA Universidad
}

\author{
Internationalization in Higher Education Institutions, \\ UNITESBA University case
}

\author{
Patricia Troncoso, \\ ORCID : 0000-0003-090I-0363 \\ Universidad UNITESBA \\ patriciatroncoso@unitesba.edu.mx \\ Carmen King, \\ ORCID : 0000-000I-8857-I42X \\ Universidad de Arizona \\ carmenking@arizona.edu
}

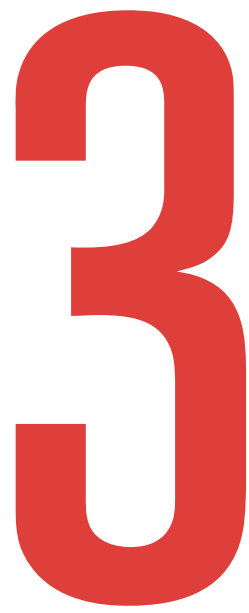

\section{Resumen}

Este estudio surge ante la necesidad de ofrecer a los estudiantes experiencias de internacionalización en casa. Describe el diseño e implementación del método de aprendizaje colaborativo internacional en línea entre la Universidad de Arizona y UNITESBA. El proceso demostró que es posible brindar experiencias de internacionalización desde casa y formación de una ciudadanía global. Algunos resultados fueron: $86 \%$ manifestó percepción positiva, $42 \%$ comunicación fluida, $21 \%$ indiferencia.

Palabras clave: Internacionalización, COIL, intercambio en línea, aprendizaje activo, ciudadanos globales.

\section{Abstract}

This study arises from the need to offer students internationalization experiences at home. Describes the design and implementation of the collaborative online international learning method between University of Arizona anda UNITESBA. The process demostrated that it is posible to provide internacionalization experiences from home and the formation of a global citizenship. Some results were: $86 \%$ manifested positive perception, $42 \%$ fluent communication, $21 \%$ indifference.

Key words: Internationalization, COIL, online Exchange, active learning, global citizens 
En el mundo actual se identifica la necesidad de forma estudiantes capaces de desarrollar su profesión en un ambiente multicultural. De allí que las Instituciones de Educación Superior (IES) estén avocadas a la internacionalización de sus salones de clase y comunidad a través de diferentes iniciativas o con una combinación de ellas.

Las IES, de acuerdo con la literatura, reconocen que la internacionalización no es una opción sino una de las habilidades que necesitan los egresados para tener acceso al mercado laboral en el contexto actual.

La literatura reconoce que estas habilidades, de internacionalización, son difíciles de enseñar en un salón de clase tradicional (Appih-Kubi, 2020), las aproximaciones más exitosas han sido a través de experiencias de aprendizaje con otras culturas con la movilidad.

En este sentido, el método tradicional que se utiliza consiste en el traslado de estudiantes a otras IES fuera del país, lo cual involucra una inversión que dejan fuera a una parte importante de la población estudiantil de cualquier Universidad del mundo (se considera que el $2 \%$ de los estudiantes en el mundo puede hacerlo). Otra propuesta, en auge, es el Collaborative Online International Learning (COIL) o aprendizaje colaborativo internacional en línea que comenzó en Europa (Prieto, 2015) y universidades como la de Nueva York (SUNY) desde hace décadas es un impulsor del método.

Este estudio describe la aplicación de esta estrategia de internacionalización, COIL, en UNITESBA Universidad una IES del Bajío mexicano. La internacionalización para esta institución va más allá de la movilidad y los convenios de colaboración, se pretende lograr la internacionalización de los estudios en la búsqueda de una mayor calidad y relevancia de la educación que se imparte. Todo ello con el propósito de lograr la competitividad de la educación, es decir, alta calidad y prestigio.

Este objetivo de internacionalización van muy relacionado con el propósito de contribuir a la formación de ciudadanos para el mundo, de acuerdo con King la estrategia de aprendizaje colaborativo en línea (COIL) es una de las mejores opciones para impactar a la mayor cantidad de estudiantes y lograr que la identidad trascienda las fronteras geográficas para establecer una comunicación entre culturas que contribuya al desarrollo del pensamiento crítico, análisis de información, empatía, trabajo colaborativo y un enfoque más amplio en la búsqueda de soluciones comunes a problemas internacionales (King, 2019). 


\section{¿QUÉ ES COIL?}

COIL es método de enseñanza relativamente nuevo y de costo accesible para promover la internacionalización a través de la colaboración entre IES y estudiantes en diferentes países, en donde los profesores involucrados diseñan actividades conjuntas, proyectos y discusiones para los estudiantes (Global Learning Conference, 2017).

De esta manera, COIL se ha vuelto una herramienta para construir las competencias de interculturalidad, una "inteligencia global" en la educación superior de México y el mundo (COIL connect, 202I). Adicionalmente, la contingencia sanitaria del 2020 (pandemia por el covid-19) potencializo el uso de esta metodología entre las IES no solo para experiencias de aprendizaje internacional, también entre IES del mismo país.

El método consiste en incluir un módulo, incluso puede ser todo un ciclo académico, a programas de estudio ya existentes en la universidad y con ello enriquecer la clase con la integración de académicos y estudiantes extranjeros, es decir, se crea una experiencia intercultural, "traer aire fresco de fuera". (Dooly 2008) (King, 2019) el proyecto puede ser de comunicación Intercultural hasta la formulación de soluciones interdisciplinarias a problemas globales.

Uno de los desafíos para llevar a cabo esta metodología es contar con una red de contactos, de académicos y universidades, para diseñar la experiencia de aprendizaje e impactar en la comunidad; en este sentido en México se creó una red que se ha propuesto difundir la metodología entre las IES de los países latinoamericanos principalmente ya que la evidencia empírica muestra el magro progreso y se vislumbra un gran potencial (LatAm COIL, 2020).

\section{LA RED LATINOAMERICANA COIL (RLC)}

La RLC es una organización liderada por cuatro universidades con I 36 IES y 200 académicos miembros (LatAm COIL, 2020), sus objetivos son promover la metodología COIL, ser un espacio de comunicación y fomentar la investigación. Dentro de ella hay IES y académicos con experiencia en la metodología y otros noveles, se ofrece a los agremiados talleres y orientación sobre la implementación de la metodología desde una perspectiva global y latinoamericana al mismo tiempo.

\section{CIUDADANÍA GLOBAL}

El termino se define como ser parte de una comunidad mundial que implica compartir valores; una identidad global que, dado el progreso tecnológico, es posible en el siglo XXI. Adicionalmente, se acepta que el mundo es complejo, conectado e interdependiente y que las acciones de los seres humanos tienen repercusiones a nivel local y mundial (Goren, 2017).

En las IES se admite que las barreras físicas y comunicativas se han queda atrás, en muchos casos, se percibe en los estudiantes una mayor demanda de compromiso global, un sentido de pertenencia a una comunidad mayor. En los salones de clase los estudiantes abordan, en diferentes asignaturas, problemas internacionales a través de métodos de aprendizaje activos como debates, juegos de rol que los forman para la vida personal y profesional en la complejidad del mundo de hoy (Chiba, 202I).

El resultado esperado de este aprendizaje es formar estudiantes seguros de sí, ciudadanos responsables y colaborativos, a través de actividades que les permiten explorar, desarrollar y expresar sus propios valores y opiniones, al tiempo que desarrollan habilidades de escucha y respeto hacia los otros y sus puntos de vista, con lo cual se espera que podrán tomar decisiones informadas que les impactarán a ellos y a los demás. 


\section{UMR: MANAGEMENT REVIEW}

\section{CASO DE ESTUDIO}

UNITESBA Universidad es una IES con más de 30 años que imparte educación del nivel básico al posgrado ubicada en Celaya, Guanajuato, México.

Con motivo de la implementación de esta estrategia (COIL) se llevó a cabo una revisión curricular y metodológica de los programas formativos guiada por la ambición de preparar mejor a los estudiantes para un mercado laboral global, ya que cuando se habla de internacionalización de los estudios, se refiere a diferentes factores que impactaran en la construcción de ése rasgo de egreso. Algunos de estos elementos son: el contenido de los programas de las materias, la introducción de aspectos internacionales e interculturales y de idiomas en el desarrollo de las sesiones de clase; actividades que están a cargo del académico - mentor. En cuanto a los servicios de apoyo se ofrece una biblioteca digital y una coordinación de vinculación con opciones de movilidad y convenios nacionales e internacionales.

Figura I: Esquema de planificación de COIL

\begin{tabular}{|c|c|c|}
\hline Meta & Objetivo & Fines \\
\hline $\begin{array}{c}\text { Formación del rasgo de egreso, } \\
\text { liderazgo, inteligencia global }\end{array}$ & $\begin{array}{c}\text { Incrementar el número de estudiantes } \\
\text { con experiencia de internacionaliza- } \\
\text { ción, a través del método COIL }\end{array}$ & $\begin{array}{c}\text { Misión, Visión de } \\
\text { UNITESBA Universidad }\end{array}$ \\
\hline
\end{tabular}

Elaboración propia 2020

La incorporación de esta estrategia de internacionalización comenzó con la membresía de UNITESBA Universidad a la naciente Red Latinoamericana COIL para aplicar "internacionalization at home" es decir actividades de internacionalización en casa o desde casa y con ello que un número mayor de estudiantes pudiera desarrollar esta habilidad, y no solo quienes pueden participar en programas de movilidad.

El objetivo planteado para el intercambio online fue que los estudiantes se relacionaran de manera eficaz, se comunicarán y seleccionaran una idea internacional de negocio. Los resultados del aprendizaje esperados fueron: desarrollar las habilidades lingüísticas e interculturales, a través del desarrollo de un proyecto que les permitiera usar la lengua con propósitos comunicativos reales, además de la comunicación intercultural, uso de TIC y aprender a aprender.

En la figura dos se muestra el modelo de implementación.

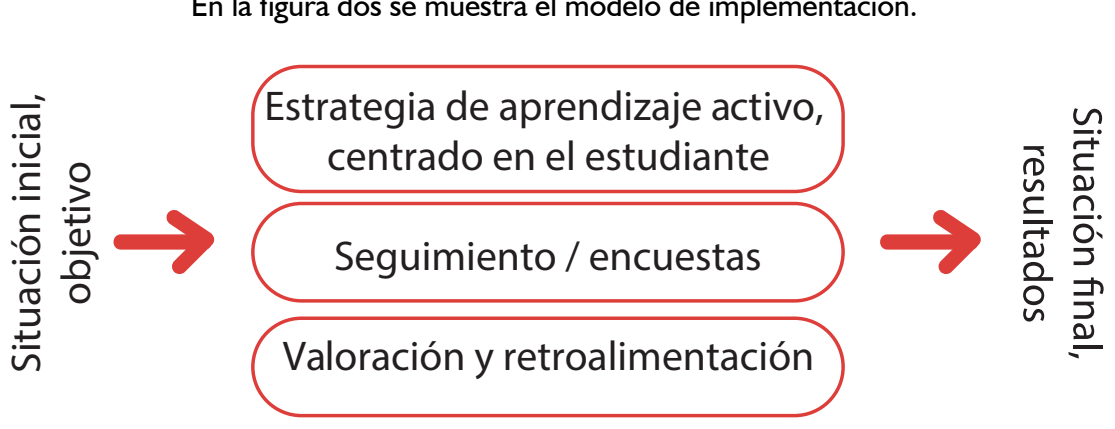

Elaboración propia 2020

El modelo establece valorar la situación inicial y formular el objetivo del intercambio en particular. Durante el proceso aplicar una estrategia de aprendizaje activo, centrado en el estudiante que implica: hablar, discutir, hacer, practicar; de manera continua dar seguimiento, levantar encuestas de opinión y retroalimento a los estudiantes e incorporar mejoras. Finalmente valorar el nivel de satisfacción de los participantes y el cumplimiento de los objetivos. 
El resultado obtenido por la empresa "El Campeón" en el diagnóstico previo, vía el cuestionario de Luna, Castillo y Romero A continuación, se muestra un esquema más detallado del proceso en tres etapas para la implementación de la experiencia de aprendizaje colaborativo internacional en línea, ver figura 2:

Figura 3 Implementación, COIL

\begin{tabular}{|c|c|c|c|c|c|}
\hline Continuo & $\begin{array}{l}\text { redes de } \\
\text { colaboración }\end{array}$ & $\begin{array}{l}\text { encontrar un } \\
\text { socio }\end{array}$ & capacitar académicos & & \\
\hline Inicio & $\begin{array}{l}\text { diseñar la expe- } \\
\text { riencia }\end{array}$ & $\begin{array}{l}\text { sensibilizar a los } \\
\text { estudiantes }\end{array}$ & $\begin{array}{l}\text { formar equipos mez- } \\
\text { clando estudiantes } \\
\text { locales e invitados }\end{array}$ & $\begin{array}{l}\text { encuesta previa } \\
\text { al desarrollo de } \\
\text { la experiencia }\end{array}$ & \\
\hline \multirow[b]{2}{*}{ Desarrollo } & $\begin{array}{l}\text { inicio en reunión } \\
\text { sincrónica, expli- } \\
\text { cación del plan } \\
\text { de trabajo }\end{array}$ & $\begin{array}{l}\text { actividades de so- } \\
\text { cialización entre } \\
\text { estudiantes }\end{array}$ & $\begin{array}{l}\text { organización entre } \\
\text { estudiantes para el } \\
\text { desarrollo del pro- } \\
\text { yecto }\end{array}$ & $\begin{array}{l}\text { reuniones sin- } \\
\text { crónicas entre } \\
\text { equipos de } \\
\text { estudiantes }\end{array}$ & $\begin{array}{l}\text { presentación } \\
\text { de avances en } \\
\text { reunión grupal }\end{array}$ \\
\hline & & & $\begin{array}{l}\text { asesoría de acadé- } \\
\text { micos y retroalimen- } \\
\text { tación, } \\
\text { implementar mejo- } \\
\text { ras, acompañar }\end{array}$ & $\begin{array}{l}\text { encuesta inter- } \\
\text { media ¿cómo } \\
\text { va el proyecto? } \\
\text { ¿qué retos han } \\
\text { enfrentado? }\end{array}$ & \\
\hline
\end{tabular}

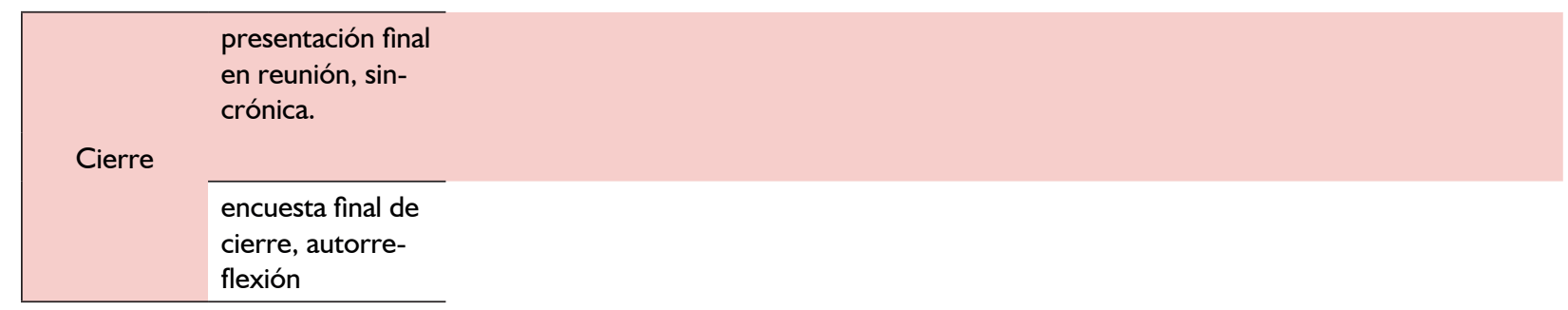

Elaboración propia 2021

En el primer semestre del 202I, se trazó la experiencia de enseñanza y aprendizaje mediada por el uso de internet y llevada a cabo por la Ph. D. Carmen King de University of Arizona, USA y Mtra. Patricia C. Troncoso de UNITESBA Universidad, México con una duración de siete semanas.

En el intercambio en línea participaron 16 estudiantes de pregrado, de University of Arizona (UA), con un promedio de edad 20 años, nivel de español intermedio BI de la lengua extranjera/segunda lengua conforme al Marco Común Europeo de Referencia (MCER) (Consejo de Europa, 2002), yl4 estudiantes de pregrado de UNITESBA Universidad, de la misma edad. El proyecto se desarrolló en idioma español en la plataforma teams de Microsoft y con el uso optativo y ocasional de WhatsApp, e Instagram.

De acuerdo con un sondeo de opinión y encuesta previa al inicio los estudiantes dijeron sentir: ansiedad (7I.42\%), nerviosismo (42.85\%) e inseguridad por enfrentarse a una situación nueva (28.57\%); carencias culturales (desconocimiento de la contraparte) (92.85\%); carencias léxicas (vocabulario básico, escasez de recursos conversacionales) $(78.57 \%)$

La estrategia de aprendizaje activa que se utilizó fue el aprendizaje por proyectos (APP), que es un tipo de aprendizaje conectado al mundo real, en donde los estudiantes desarrollan habilidades tales como la resolución de problemas, pensamiento crítico y trabajo en equipo. 


\section{UMR: MANAGEMENT REVIEW}

Durante el módulo COIL se planearon una serie de actividades simples y accesibles de interacción. Se utilizo la plataforma teams de Microsoft que permite el uso de chats, correo, fotos, videoconferencias, también se utilizó ZOOM para reuniones generales. Se crearon equipos para expresar ideas y puntos de vista; se estableció un cronograma de tareas y revisión periódica sobre el sentir, participación y desarrollo de las actividades.

En la valoración intermedia, se encontró que un $26.66 \%$ de los estudiantes no participaba en las actividades, por dificultades con la tecnología $6.66 \%$ y un $20 \%$ por situaciones personales, éstos últimos abandonaron el curso en su Universidad y en consecuencia el intercambio online por motivos estrictamente privados.

Sin embargo, en el resto de los estudiantes se encontró que el $42.10 \%$ dijo estar altamente motivado, el $36 \%$ preocupado por no poder hacerlo bien (las actividades) y un $21.05 \%$ indiferente.

En el aspecto de la comunicación durante las reuniones, el $42.10 \%$ dijo que era fluida y sin complicaciones, la misma proporción señaló que resolvieron los problemas que se presentaron $y$, un $10.52 \%$ manifestó que no tenía comunicación.

Frente a la cuestión, ¿qué puedes realizar para hacer más enriquecedora la experiencia del intercambio? algunas de las respuestas fueron:
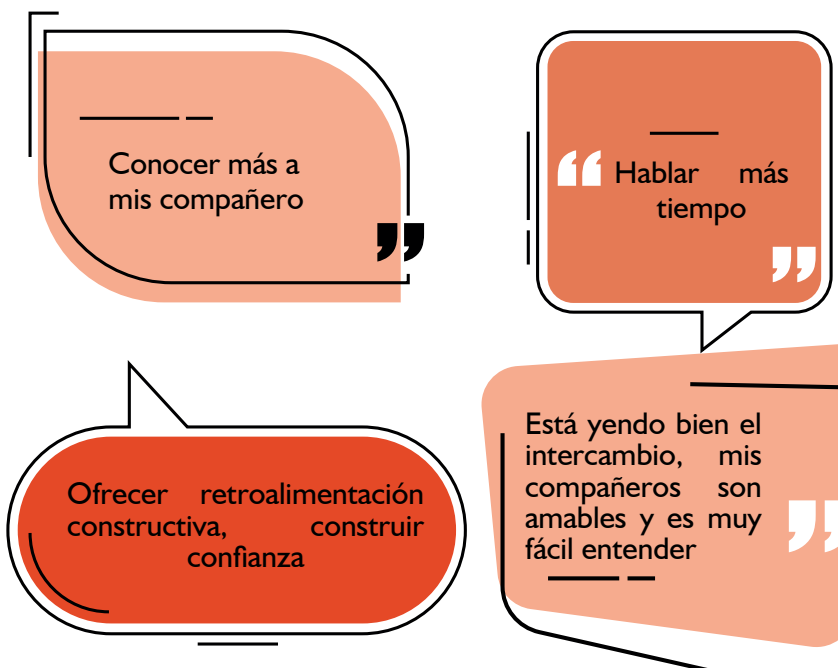

Está yendo bien el intercambio, mis compañeros son amables y es muy fácil entender
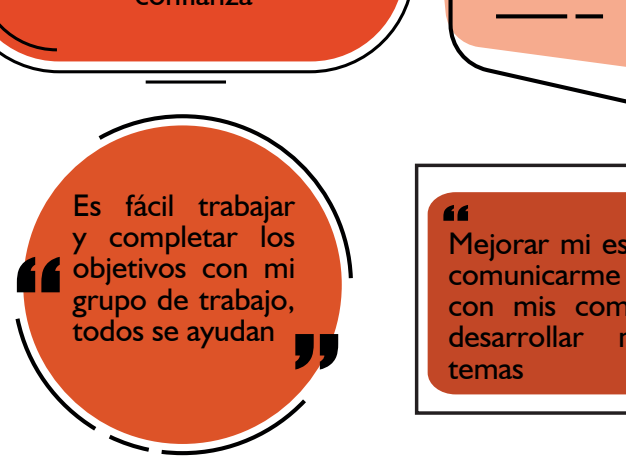

El reto para los estudiantes fue conocer la relación que hay entre estos países vecinos, comercial y cultural, y desarrollar una idea de negocio internacional. Para ello contaron con algunos materiales: lecturas, vídeo y un micro taller. Los equipos llevaron a cabo tareas y principalmente explorar, ideas y construir su propuesta. La presentación final consistió en la elaboración de un vídeo en donde explicaban su idea de negocio.

Para dar por concluida la experiencia se aplicó una encuesta de autorreflexión para intercambios internacionales de la Universidad de Arizona, un instrumento de tres dimensiones y 23 ítems con una escala de tres niveles que fueron: Necesito trabajar/ tengo dificultades; ejecutándolo/ puedo hacerlo, y sobresaliente/ soy muy bueno; en el siguiente apartado, se muestran los ítems y el promedio.

\section{PLANIFICACIÓN Y ASISTENCIA A REUNIONES}

En la dimensión de planificación y asistencia a reuniones el promedio fue de 2.4868 , la ponderación más baja de las tres dimensiones, pero significa ejecutándolo/ puedo hacerlo, la siguiente tabla muestra las unidades mínimas de medida:

Tabla I: Autorreflexión para intercambios internacionales, Planificación y asistencia a reuniones

Planificación y Asistencia a reuniones

Estoy al día con el calendario de tareas y las fechas de entrega.

Ayudo a planificar las reuniones

Desarrollo una agenda para todas las reuniones.

Asisto a todas las reuniones.

Durante las reuniones, aporto ideas originales y comento sobre las ideas de mis compañeros.

Aviso con anticipación si no podré asistir a una junta o completar alguna tarea.

Busco maneras de comunicarme con mi equipo cuando experimento problemas tecnológicos.

Muestro flexibilidad con cambios de horarios e inconvenientes que se presenten.

Respondo de manera rápida (dentro de 24 horas) a los correos y mensajes que me manden mis compañeros.

Fuente: UA 2020 


\section{COMUNICACIÓN CON LOS COMPAÑEROS}

La dimensión de comunicación con los compañeros mostró un promedio de 2.8639 , con lo cual se demostró que uno de los principales objetivos se cumplió: la comunicación en un contexto real entre culturas diferentes, pero con un propósito en común.

Tabla 2: Autorreflexión para intercambios internacionales, Comunicación con los compañeros

\begin{tabular}{|l|}
\hline \multicolumn{1}{|c|}{ Comunicación con los compañeros } \\
\hline Soy respetuoso y considero las opiniones de todos mis compañeros. \\
\hline Les repito a mis compañeros lo que he entendido de la conversación para evitar confusiones. \\
\hline Demuestro tolerancia ante opiniones distintas a las mías. \\
\hline Trato de comunicarme en el idioma que estudio y/o el idioma de mis compañeros. \\
\hline Modifico mi conducta para crear un ambiente grupal positivo. \\
\hline Pido aclaraciones cuando no entiendo una idea o palabra. \\
\hline Ayudo a mis compañeros que batallen con comunicar sus ideas. \\
\hline
\end{tabular}

Fuente: UA 2020

\section{PROYECTO COLABORATIVO}

La última dimensión evaluada sobre el proyecto colaborativo indicó un 2.6607 que significa puedo hacerlo. Durante el intercambio todos los equipos superaron las dificultades y entregaron el proyecto final en tiempo y forma.

Tabla 3: Autorreflexión para intercambios internacionales, Proyecto colaborativo.

\begin{tabular}{|l|}
\hline \multicolumn{1}{|c|}{ Proyecto Colaborativo } \\
\hline Entiendo las instrucciones de las tareas y las fechas finales \\
\hline Pido aclaraciones cuando no entiendo una tarea. \\
\hline Participe activamente en la plantificación de proyectos grupales y tareas. \\
\hline Ayudo a que las opiniones de todos se vean integradas en el proyecto grupal. \\
\hline Mantengo un contacto frecuente con mis compañeros grupales. \\
\hline Conozco y cumplo con mis responsabilidades en el proyecto grupal \\
\hline Revise la parte del proyecto que hicieron mis compañeros, para poder darles comentarios. \\
\hline
\end{tabular}

Fuente: UA 2020

Uno de los hallazgos fue una fuerte correlación entre las variables: vIDurante las reuniones, aporto ideas originales y comento sobre las ideas de mis compañeros y v3 Ayudo a que las opiniones de todos se vean integradas en el proyecto grupal, que se muestra en la siguiente figura:

Figura 4: Correlación positiva entre Aporto ideas .. y

Ayudo a que las opiniones de todos se vean integradas al proyecto.

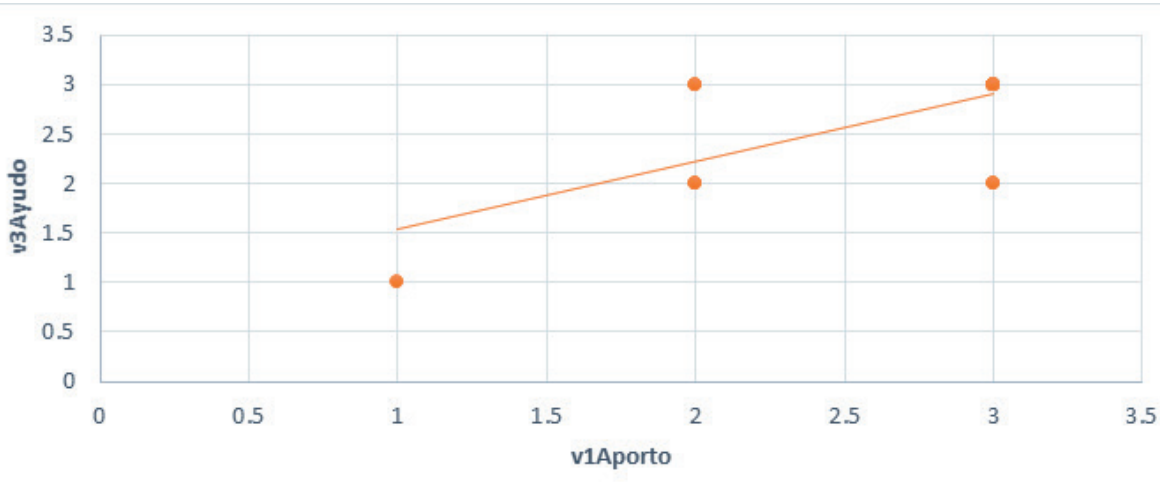

Fuente: Elaboración propia 2020 
Otro resultado relevante fue que la variable: Modifico mi conducta para crear un ambiente grupal positivo, representó la mayor proporción de las respuestas de esa dimensión, ver figura:

Figura 5: Modifico mi conducta.

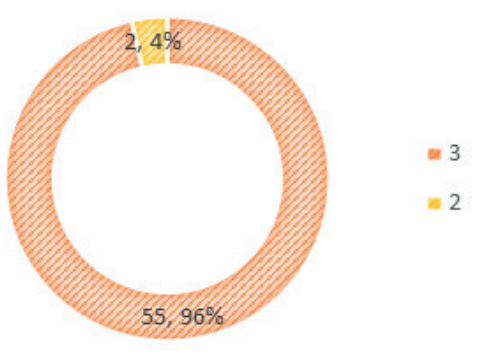

Elaboración propia 2020

La variable: Conozco y cumplo con mis responsabilidades en el proyecto grupal también fue uno de los resultados relevantes que se encontraron. Este ítem se relaciona con la medición de la ética, integridad, ver figura 5:

Figura 6: v Conozco y cumplo con mis responsabilidades en el proyecto grupal.

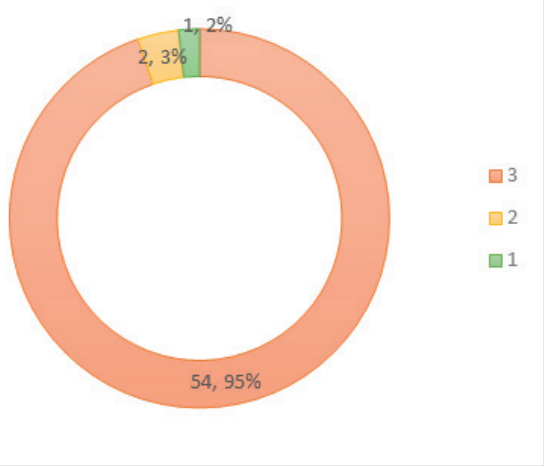

Elaboración propia 2020

La percepción de los estudiantes de la experiencia fue positiva (86\%) en una escala de uno a tres la percepción general fue de 2.6705

Figura 7: Percepción general de los estudiantes del intercambio online

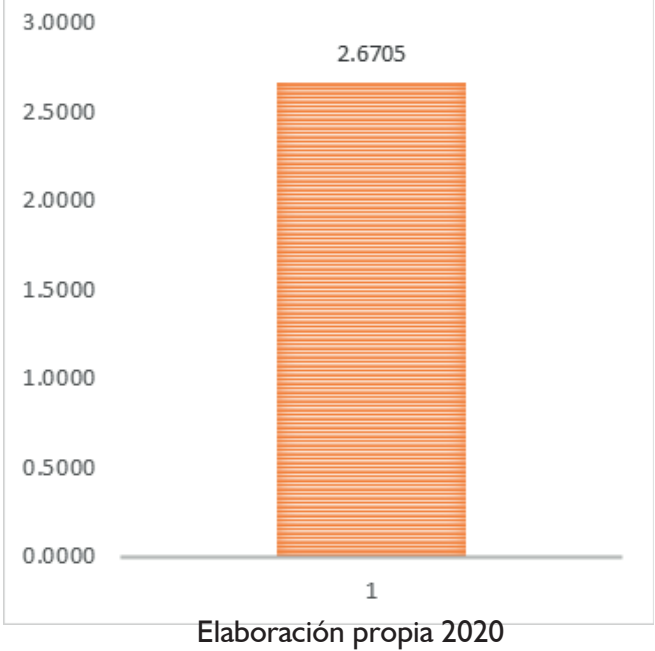


El proyecto logro su objetivo con el desarrollo del proceso de implementación de una estrategia más de internacionalización para la IES objeto de estudio. La experiencia de aprendizaje entre AU y UNITESBA también logro la meta planteada, ya que se desarrollaron las competencia lingüísticas e interculturales, a través del desarrollo de un proyecto que les permitió usar la lengua con propósitos comunicativos reales.

El $86 \%$ de los estudiantes participantes observó un desempeño positivo en el nivel de responsabilidad, conducta entre pares, aportación de ideas y ayuda a que las opiniones de sus compañeros fueran escuchadas. Con ello se demostró que esta actividad le proporcionó al estudiante un ambiente intercultural en línea para desarrollar esta habilidad.

El aprendizaje activo, es decir, un enfoque interactivo para aprender o aprender haciendo, se manifestó en la colaboración entre los estudiantes para el análisis y resolución del problema que planteado y que se resolvió desde sus específicos ámbitos de conocimiento compartiendo con ello conocimientos disciplinares y generando propuestas desde una perspectiva multicultural; con ello ésta actividad también fue una acción estratégica para la internacionalización del currículo que permite situar la práctica educativa en el contexto internacional y multicultural permitiendo a los estudiantes el acceso a la internacionalización en casa.

Adicionalmente, se considera que este proyecto también contribuyó a la formación de ciudadanos globales, a mejorar la práctica educativa con el uso de tecnología para crear un ambiente de aprendizaje internacional que inspiró la innovación y mejora de la intervención docente,

\section{RECOMENDACIONES-}

El reto que se plantea es:

- desarrollar oportunidades educativas, localizando, contactando y estableciendo vínculos con profesores e IES extranjeras interesadas en colaborar en proyectos académicos internacionales en línea.

- uso de metodologías activas,

- diseño de proyectos interdisciplinarios o multidisciplinarios,

- involucrar a toda la IES para fortalecer la actividad 


\section{FUENTES BIBLIOGRÁFICAS}

Appih-Kubi, P. (2020). Global learning. International Journal of Engineering Pedagogy, 109- 124.

Chiba, M. S. (202I). Investigating effective teaching and learning for sustainable development and global citizenship: implication from a systematic review of the literature. International Journal of Educational Development.

COIL connect. (0I de 0 I de 202 I). COIL connect. Obtenido de COIL connect: https://coilconnect.org/

Consejo de Europa. (2002). Marco Común Europeo de Referencia para las Lenguas: aprendizaje, enseñanza. Madrid: MECD Anaya.

Global Learning Conference. (2017). Global learning conference. NY: Global leraning conference org.

Goren, H. Y. (2017). Global citizenship education redefined - A systematic review of empirical studies on global citizenship education. International Journal od Education Research, I70- 183.

King, C. (2019). Global citizenship education through collaborative online international learning in the borderlands: a case of the Arizona - Sonora Megaregión. Journal os Studies in International Education, 83-99.

King, C. (2020). Influences of academic culture in collaborative online international learning (COIL): differences in Mexican and US student's reported experiences. Foreing language annals, I I- 20.

LatAm COIL. (0I de 0 I de 2020). Red Latinoamericana COIL. Obtenido de Red Latinoamericana COIL: https://www. uv.mx/coil/

Prieto, L. V.-M. (2015). Internacionalización en casa en la educación superior: los retos de Colombia. Revista Internacional de Cooperación y Desarrollo. 
Derechos de Autores (202I) Patricia Troncoso y Carmen King,

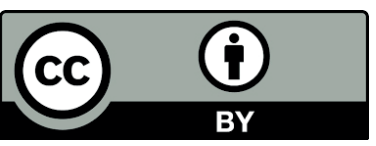

Este texto está protegido por la licencia Creative Commons 4.0.

Usted es libre de Compartir - copiar y redistribuir el material en cualquier medio o formato- y Adaptar el documento -remezcla, transformar y crear a partir del material- para cualquier propósito, incluso para fines comerciales, siempre que cumpla la condición de:

Atribución: Usted debe dar crédito a la obra original de manera adecuada, proporcionar un enlace a la licencia, e indicar si se han realizado cambios. Puede hacerlo en cualquier forma razonable, pero no de forma tal que sugiera que tiene el apoyo del licenciante o lo recibe por el uso que hace de la obra.

Resumenendelicencia - Textocompretodelalicencia 